2011

\title{
Scaling of the magnetic reconnection rate with symmetric shear flow
}

\author{
P. A. Cassak
}

A. Otto

Follow this and additional works at: https://researchrepository.wvu.edu/faculty_publications

\section{Digital Commons Citation}

Cassak, P. A. and Otto, A., "Scaling of the magnetic reconnection rate with symmetric shear flow" (2011). Faculty Scholarship. 320.

https://researchrepository.wvu.edu/faculty_publications/320

This Article is brought to you for free and open access by The Research Repository @ WVU. It has been accepted for inclusion in Faculty Scholarship by an authorized administrator of The Research Repository@ WVU. For more information, please contact ian.harmon@mail.wvu.edu. 


\title{
Scaling of the magnetic reconnection rate with symmetric shear flow
}

\author{
P. A. Cassak ${ }^{1}$ and A. Otto ${ }^{2}$ \\ ${ }^{1}$ Department of Physics, West Virginia University, Morgantown, West Virginia 26506, USA \\ ${ }^{2}$ Geophysical Institute, University of Alaska-Fairbanks, Fairbanks, Alaska 99775, USA
}

(Received 10 March 2011; accepted 20 June 2011; published online 18 July 2011)

\begin{abstract}
The scaling of the reconnection rate during (fast) Hall magnetic reconnection in the presence of an oppositely directed bulk shear flow parallel to the reconnecting magnetic field is studied using two-dimensional numerical simulations of Hall reconnection with two different codes. Previous studies noted that the reconnection rate falls with increasing flow speed and shuts off entirely for super-Alfvénic flow, but no quantitative expression for the reconnection rate in sub-Alfvénic shear flows is known. An expression for the scaling of the reconnection rate is presented. (C) 2011 American Institute of Physics. [doi:10.1063/1.3609771]
\end{abstract}

Magnetic reconnection occurs when a change in topology in a plasma's magnetic field converts magnetic energy into kinetic and thermal energy. Early attempts to model magnetic reconnection ${ }^{1,2}$ assumed a high degree of symmetry to make the problem tractable. However, these symmetries are not appropriate for almost all physical applications. In particular, the breakdown of perfect symmetries involves asymmetries in magnetic field strength and density, ${ }^{3,4}$ the presence of a bulk shear flow, ${ }^{5}$ and three-dimensional effects (see Ref. 6 for a recent review). Such complications occur in tokamaks $^{7-9}$ and many locations in the magnetosphere.

Here, we consider the effect of a shear flow, meaning a bulk flow parallel to the direction of the reconnecting magnetic field. The canonical example of this is at the dayside magnetopause. Unless reconnection occurs near the subsolar point, the solar wind introduces a component of flow along the reconnecting field. This is most pronounced at the cusps when the interplanetary magnetic field (IMF) is northward. Early observations ${ }^{5,10}$ demonstrated the existence of these flows, and they are often sub-Alfvénic. Shear flows are now routinely observed. ${ }^{1-20}$ Statistical studies showed that reconnection at the cusp occurs $90 \%$ of the time when the IMF is northward $^{21}$ and the shear flow speed is sub-Alfvénic. ${ }^{22-24}$

In the present discussion, we address the effect of shear flow on reconnection, meaning the scaling of reconnection parameters (the functional dependence of reconnection properties such as the reconnection rate on system parameters). Much work has been done on the theory of reconnection with a shear flow; we omit a discussion of the effect on the shock structure of the outflow region because we are focused on scaling. The first large-scale simulations study ${ }^{25}$ employed two-dimensional magnetohydrodynamics (MHD) with a localized resistivity. They showed that reconnection does not occur if the flow speed is greater than the Alfvén speed, which agrees with the previous theoretical work. ${ }^{26}$ (Interestingly, there is a strong analogy between this and the results showing that reconnection is suppressed when the diamagnetic drift is super-Alfvénic, ${ }^{27}$ which has been confirmed by observations in the solar wind. ${ }^{28}$ ) When the flow is sub-Alfvénic, the dissipation region gets twisted and the opening angle is larger. A follow-up paper ${ }^{29}$ included the effects of an asymmetric density, showing that the two effects can compete or enhance each other depending on parameters.

More recently, it was shown that the reconnection rate decreases as a function of increasing shear flow, ${ }^{30,31}$ though no quantitative prediction was presented. Interestingly, while the linear tearing and Kelvin-Helmholtz instabilities are unstable in different parameter regimes in MHD, both instabilities can be linearly unstable for the same parameters in Hall-MHD (Ref. 32) because ions decouple from electrons near the current layer. Hall-MHD simulations were used to show that shear flow causes flow vortices in magnetic islands during asymmetric reconnection. ${ }^{33}$ The linear phase of reconnection with a shear flow was studied in particle-in-cell simulations $^{34}$ and MHD simulations addressed the effect of the thickness of the shear flow layer. ${ }^{31}$ Particle-in-cell simulations addressed the combined effects of asymmetries, a guide field, and shear flow. ${ }^{35}$ Simulations of reconnection with a unidirectional flow were also studied recently. ${ }^{36}$

This paper seeks to obtain a quantitative prediction for the scaling of the reconnection rate as a function of shear flow speed for sub-Alfvénic flow. Simulations of Hall reconnection with varying shear flow speed keeping all else constant are presented using two independent codes (with different boundary conditions and dissipation mechanisms). The result is that both codes reveal the same quantitative result. We find that the magnetic field upstream of the dissipation region self-consistently changes systematically in the presence of a shear flow. The effect of this on the rate of reconnection is discussed. Further properties of reconnection with a symmetric shear flow are discussed in a follow-up paper. $^{37}$

The geometry of the system under consideration is sketched in Fig. 1. The shear flow is initially parallel to the reconnecting magnetic field. We assume the system is twodimensional with no initial out-of-plane (guide) magnetic field. For simplicity, we do not consider any asymmetries meaning that the fields, densities, temperatures, and shear flow speeds are equal on either side of the dissipation region. The upstream magnetic field has strength $B$ and the magnitude of the shear flow is $v_{s}$. 


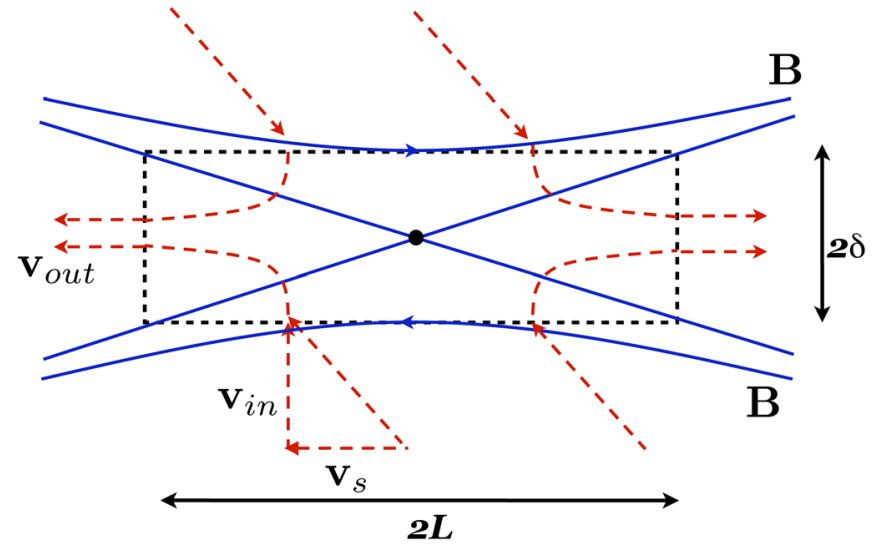

FIG. 1. (Color online) Schematic diagram of the reconnection region in the presence of a shear flow. Magnetic field lines are (blue) solid lines and velocity flow are (red) dashed lines.

Two-dimensional simulations of compressible Hall reconnection with a shear flow are performed using two independent codes, F3D (Ref. 38) and HALL2 (Ref. 39). In both, the density, ion velocity, magnetic field, and pressure are evolved in time. Both normalize magnetic fields, number densities, velocities, lengths, electric fields and pressures to $B_{0}, n_{0}$, the Alfvén speed $c_{A 0}=B_{0} /\left(4 \pi m_{i} n_{0}\right)^{1 / 2}$, the ion inertial length $d_{i 0}=\left(m_{i} c^{2} / 4 \pi n_{0} e^{2}\right)^{1 / 2}, \quad E_{0}=c_{A 0} B_{0} / c$, and $P_{0}=B_{0}^{2} / 4 \pi$, respectively.

We describe the F3D simulations first. Variables are evolved explicitly using the trapezoidal leapfrog in time and fourth order finite difference in space. The computational domain has a size $L_{x} \times L_{y}=204.8 \times 102.4$ with a cell size of $0.05 \times 0.05$ and uses periodic boundary conditions in both directions. To test the resolution, a run is performed at double the resolution during the steady-state phase and the results are unchanged. The initial magnetic field profile is a double tearing mode

$$
B_{x}(y)=\tanh \left(\frac{y-L_{y} / 4}{w_{0}}\right)-\tanh \left(\frac{y-L_{y} / 4}{w_{0}}\right)-1,
$$

where $w_{0}=1.0$ is the initial current sheet thickness. The initial density profile is uniform with $n=1$. The temperature is initially nonuniform to balance total pressure with an asymptotic value of 1 . The initial shear flow profile is analogous to the magnetic field profile

$$
v_{x}(y)=v_{s}\left[\tanh \left(\frac{y-L_{y} / 4}{w_{0}}\right)-\tanh \left(\frac{y-L_{y} / 4}{w_{0}}\right)-1\right],
$$

where we assume the same thickness $w_{0}$ for the flow profile, but this is not a necessary condition. ${ }^{31}$

Reconnection is initiated using a magnetic field perturbation of $\delta \mathbf{B}=-\left(0.012 L_{y} / 2 \pi\right) \widehat{\mathbf{z}} \times \nabla\left[\sin \left(2 \pi x / L_{x}\right)\right.$ $\left.\sin ^{2}\left(2 \pi y / L_{y}\right)\right]$. The ratio of specific heats $\gamma$ is $5 / 3$. There is no viscosity or resistivity, but fourth order diffusion with coefficient $5 \times 10^{-5}$ is used in all equations to damp noise at the grid scale. Initial random perturbations on the magnetic field of amplitude 0.00005 break symmetry so that secondary magnetic islands are ejected. The electron inertia is $m_{e}=m_{i} / 25$.

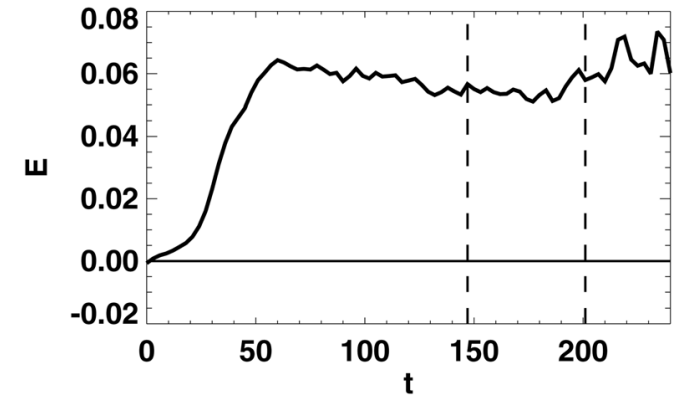

FIG. 2. Reconnection rate $E$ as a function of time $t$ for the F3D simulation with $v_{s}=0.4$. Results are averaged over the steady time between the two vertical dashed lines.

To determine the scaling of the reconnection rate, the system is evolved until transient effects have subsided and a quasi-steady state is achieved, meaning that we are in the nonlinear regime rather than the linear tearing regime. The reconnection rate $E$ is calculated as the time rate of change of the magnetic flux between the X-line and the O-line. The shear flow is varied from $v_{s}=0.0$ to 1.2 with all else held constant. A representative plot of the time evolution of $E$ is shown in Fig. 2 for $v_{s}=0.4$. The presented values of $E$ are time averages over sufficiently steady times when the magnetic island is large, denoted by the two vertical dashed lines in Fig. 2.

Results for $E$ as a function of $v_{s}$ are plotted in Fig. 3(a). First, note that there is no reconnection for $v_{s}>1$, consistent with previous results from resistive-MHD simulations. ${ }^{29}$ Second, as expected, the reconnection rate decreases with $v_{s}$. The dashed line has the dependence

$$
E=E_{0}\left(1-\frac{v_{s}^{2}}{c_{A}^{2}}\right)
$$

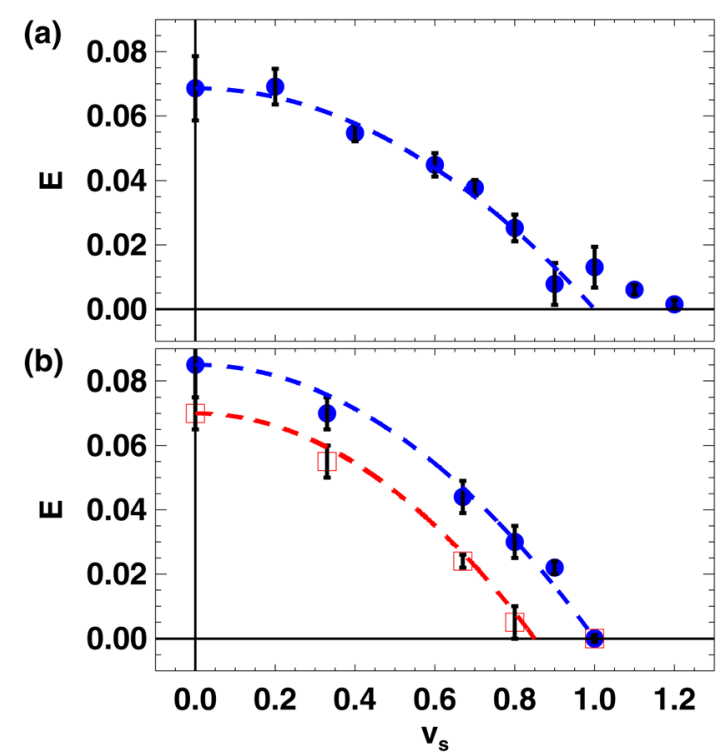

FIG. 3. (Color online) Reconnection rate $E$ as a function of shear flow $v_{s}$ for the (a) F3D and (b) HALL2 simulations. For the latter, the (blue) dots used localized $\eta$ and (red) squares used current dependent $\eta$. The dashed lines are Eq. (1). 


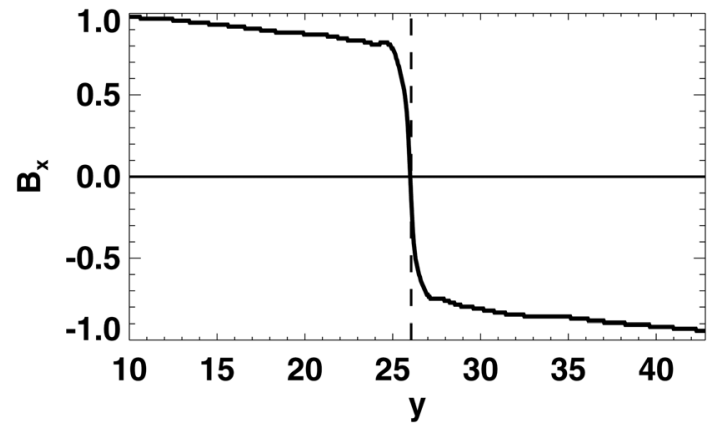

FIG. 4. Reconnecting magnetic field $B_{x}$ in a cut across the X-line in the inflow (y) direction for the F3D simulation with $v_{s}=0.4$. The vertical dashed line goes through the $\mathrm{X}$-line.

where $E_{0}$ is the measured reconnection rate in the absence of a shear flow. The agreement is very good. It is interesting to note that the form in parentheses also arises in the linear theory of the tearing mode with a shear flow, ${ }^{40}$ although the full expression for the growth rate has a more complicated dependence on $v_{s}$.

One must be careful in interpreting this result. This expression is the absolute reconnection rate, but one usually presents the reconnection rate normalized to the upstream magnetic field $B_{\text {up }}$. We find $B_{\text {up }}$ self-consistently varies as a function of shear flow. A plot of the reconnecting magnetic field strength $B_{x}$ in a cut across the X-line in the inflow direction is plotted in Fig. 4 for $v_{s}=0.4$, and one can see that the field strength $d_{i}$ upstream is noticeably less than the initial asymptotic field of 1 .

To quantify this, $B_{\text {up }}$ is measured as $B_{x}$ at the edge of the current sheet. For the ions, the edge is defined as twice the distance from the $\mathrm{X}$-line to the point where the electron and ion inflow speeds differ by $15 \%$ of the maximum ion inflow speed. For the electrons, the edge is defined as the $e$-folding distance of the out-of-plane current $J_{z}$. The results are plotted in Fig. 5 for runs with not excessively large $v_{s}$ for which a reliable $B_{\text {up }}$ was attainable. The upper (red) dots and the lower (blue) denote the upstream field for the ions and electrons, respectively. There is a systematic decrease in $B_{\text {up }}$ with $v_{s}$, which matches well with the expression

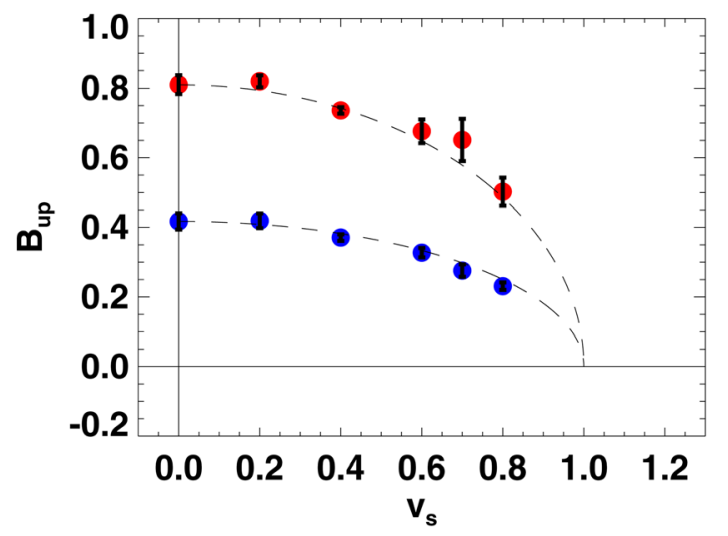

FIG. 5. (Color online) Upstream magnetic field $B_{\text {up }}$ as a function of shear flow speed $v_{s}$ for ions (top, in red) and electrons (bottom, in blue). The dashed lines are given by Eq. (2).

$$
B_{\text {up }}=B_{\text {up }, 0} \sqrt{1-\frac{v_{s}^{2}}{c_{A}^{2}}},
$$

where $B_{\text {up }, 0}$ is the upstream field when $v_{s}=0$. This is plotted as the dashed line, showing good agreement.

We now turn to the HALL2 simulations. The algorithm is second order finite difference which employs a $503 \times 128$ nonuniform grid with a maximum resolution of $\Delta x=0.2$ and $\Delta y=0.1$ in the diffusion region. The system size is $L_{x} \times L_{y}=120 \times 24$. The initial configuration is a single current sheet and initial flow with profile

$$
\begin{gathered}
B_{x}(y)=\tanh \left(y / w_{0}\right), \\
v_{x}(y)=v_{s} \tanh \left(y / w_{0}\right),
\end{gathered}
$$

with a magnetic perturbation $\delta \mathbf{B}=-0.25 \hat{\mathbf{z}} \times \nabla$ $\left[\cosh ^{-2}(x / 2) \cosh ^{-2}(y)\right]$. Boundary conditions are open (zero derivatives across all boundaries allowing for inflow through the boundaries). Results are obtained for two resistivity models, one of which uses a localized resistivity of the form $\eta(x, y)=\eta_{0} \cosh ^{-1} x \cosh ^{-1} y$ and the other a current dependent model of the form $\eta(j)=\eta_{0}\left[j^{2}-j_{c}^{2}\right]^{1 / 2}$ for $j>j_{c}$, where $\eta_{0}=0.05$.

Results for the reconnection rate are obtained using the approximate maximum reconnection rate during which the system in the vicinity of the X-line is approximately steady. Results are plotted in Fig. 3(b). The constant localized resistivity results are shown in (blue) dots and show the same behavior found in F3D. The current dependent resistivity runs are in (red) squares. One difference is that reconnection switches off in the current dependent resistivity case for shear flows larger than about 0.8 rather than 1.0. The dashed line is Eq. (1) modified to have a cutoff speed of 0.85 , which reveals that the scaling with $v_{s}$ is similar to the other simulations.

It is important to discuss normalization. The present results show that Eq. (1) gives the scaling of $E$ with $v_{s}$. To get a normalized (dimensionless) value, one typically defines $E^{\prime}=c E / B c_{A}$, but there is some freedom in choosing which magnetic field to normalize to. If one normalizes to the asymptotic field $B_{0}$, then Eq. (1) is the appropriate result. If one normalizes to $B_{\text {up }}$, Eqs. (1) and (2) imply that $E \sim E_{0}$ is independent of $v_{s}$. Note also that the (dimensional) inflow speed $v_{\text {in }}$ scales as

$$
v_{\text {in }}=v_{\text {in }, 0} \sqrt{1-\frac{v_{s}^{2}}{c_{A}^{2}}},
$$

where $v_{\text {in }, 0}$ is the inflow velocity in the absence of shear flow, where we use Eqs. (1) and (2) and $E \sim v_{\text {in }} B_{\text {up }} / c$.

The scaling of $B_{\text {up }}$ provides an explanation for the stronger dependence (and earlier stabilization) of the reconnection rate for current dependent resistivity. A smaller $B_{\text {up }}$ implies lower current densities in the diffusion region, so the current dependent resistivity is more easily switched off for larger shear flow.

In summary, we report results of a study of the scaling of the reconnection rate during collisionless reconnection with an antiparallel symmetric shear flow. The theory and simulations are restricted to two dimensions with antiparallel 
magnetic fields. The result is that the dimensional reconnection rate decreases as $1-v_{s}^{2} / c_{A}^{2}$.

To understand why the reconnection rate scales with this particular functional form, the key is to note that the shear flow decreases the efficiency of the newly reconnected field lines to drive the outflow. A decrease in outflow speed leads to a decrease in the thickness of the layer, which throttles the reconnection process. A derivation of this is the subject of a follow-up paper. ${ }^{37}$ Note, while the dissipation region for collisionless reconnection has a two-scale structure, it is the shear flow at the ion scale that controls the overall reconnection rate.

For applications to physical systems, it is necessary to extend the present result for different flow speeds on either side, which will be the subject of a future study. Additional studies should relax the simplifying assumptions of the model, such as two-dimensionality and that the magnetic fields and shear flows are parallel. A limitation of the present simulations is that the Hall-MHD model breaks down at electron scales, so confirming the results with particle-in-cell simulations would be useful.

P.A.C. thanks T. D. Phan for helpful conversations. The authors acknowledge support by NSF Grants AGS-0953463 (PAC) and AGS-0614012 (AO) and NASA Grant NNX10AN08A (PAC). Resources at the National Energy Research Scientific Computing Center were used.

${ }^{1}$ E. N. Parker, J. Geophys. Res. 62, 509, doi:10.1029/JZ062i004p00509 (1957).

${ }^{2}$ H. E. Petschek, in AAS/NASA Symposium on the Physics of Solar Flares, edited by W. N. Ness (NASA, Washington, DC, 1964), p. 425.

${ }^{3}$ R. H. Levy, H. E. Petschek, and G. L. Siscoe, AIAA J. 2, 2065 (1964).

${ }^{4}$ H. E. Petschek and R. M. Thorne, Astrophys. J. 147, 1157 (1967).

${ }^{5}$ J. T. Gosling, M. F. Thomsen, and S. J. Bame, J. Geophys. Res. 91, 3029, doi:10.1029/JA091iA03p03029 (1986).

${ }^{6}$ D. I. Pontin, Adv. Space Res. 47, 1508 (2011).

${ }^{7}$ V. V. Mirnov, C. C. Hegna, S. C. Prager, C. R. Sovinec, and H. Tian, in Proceedings of the 21st IAEA FEC, China, 16-21 October 2006 (IAEA, Vienna, Austria, 2007), pp. TH/P3-18.

${ }^{8}$ N. A. Murphy and C. R. Sovinec, Phys. Plasmas 15, 042313 (2008).

${ }^{9}$ R. J. La Haye, D. P. Brennan, R. J. Buttery, and S. P. Gerhardt, Phys. Plasmas 17, 056110 (2010).

${ }^{10}$ J. T. Gosling, M. F. Thomsen, S. J. Bame, and R. C. Elphic, J. Geophys. Res. 96, 14097, doi:10.1029/91JA01139 (1991).

${ }^{11}$ J. T. Gosling, M. F. Thomsen, G. Le, and C. T. Russell, J. Geophys. Res. 101, 24765, doi:10.1029/96JA02254 (1996).

${ }^{12}$ R. L. Kessel, S.-H. Chen, J. L. Green, S. F. Fung, S. A. Boardsen, L. C. Tan, T. E. Eastman, J. D. Craven, and L. A. Frank, Geophys. Res. Lett. 23, 583, doi:10.1029/95GL03083 (1996).

${ }^{13}$ T. G. Onsager, J. D. Scudder, M. Lockwood, and C. T. Russell, J. Geophys. Res. 106, 25467, doi:10.1029/2000JA000444 (2001).
${ }^{14}$ L. A. Avanov, V. N. Smirnov, J. H. Waite, Jr., S. A. Fuselier, and O. L. Vaisberg, J. Geophys. Res. 106, 29491, doi:10.1029/2000JA000460 (2001).

${ }^{15}$ A. Federov, E. Dubinin, P. Song, A. Skalsky, and E. Budnik, J. Geophys. Res. 106, 25419, doi:10.1029/2000JA900104 (2001).

${ }^{16}$ T. D. Phan, B. U. O. Sonnerup, and R. P. Lin, J. Geophys. Res. 106, 25489, doi:10.1029/2001JA900054 (2001).

${ }^{17}$ T. Phan, H. U. Frey, S. Frey, L. Peticolas, S. Fuselier, C. Carlson, H. Rème, J. -M. Bosqued, A. Balogh, M. Dunlop, L. Kistler, C. Mouikis, I. Dandouras, J. A. Sauvard, S. Mende, J. McFadden, G. Parks, E. Moebius, B. Klecker, G. Paschmann, M. Fujimoto, S. Petrinec, M. F. Martucci, A. Korth, and R. Lundin, Geophys. Res. Lett. 30, 1509, doi:10.1029/ 2003GL016885 (2003).

${ }^{18}$ T. D. Phan, H. Hasegawa, M. Fujimoto, M. Oieroset, T. Mukai, R. P. Lin, and W. Paterson, Geophys. Res. Lett. 33, L09104, doi:10.1029/2006GL025756 (2006).

${ }^{19}$ T. D. Phan, G. Paschmann, C. Twitty, F. S. Mozer, J. T. Gosling, J. P. Eastwood, M. Øieroset, H. Rème, and E. A. Lucek, Geophys. Res. Lett. 34, L14104, doi:10.1029/2007GL030343 (2007).

${ }^{20}$ S. Eriksson, J. T. Gosling, T. D. Phan, L. M. Blush, K. D. C. Simunac, D. Krauss-Varban, A. Szabo, J. G. Luhmann, C. T. Russell, A. B. Galvin, and M. H. Acuña, J. Geophys. Res. 114, A07103, doi:10.1029/2008JA013990 (2009).

${ }^{21}$ C. Twitty, T. D. Phan, G. Paschmann, B. Lavraud, H. Rème, and M. Dunlop, Geophys. Res. Lett. 31, L19808, doi:10.5194/angeo-22-23552004 (2004).

${ }^{22}$ B. Lavraud, T. D. Phan, M. W. Dunlop, M. G. G. T. Taylor, P. J. Cargill, J.-M. Bosqued, I. Dandouras, H. Rème, J.-A. Sauvaud, C. P. Escoubet, A. Balogh, and A. Fazakerley, Ann. Geophys. 22, 3039 (2004).

${ }^{23}$ B. Lavraud, A. Fedorov, E. Budnik, M. F. Thomsen, A. Grigoriev, P. J. Cargill, M. W. Dunlop, H. Rème, I. Dandouras, and A. Balogh, J. Geophys. Res. 110, A02209, doi:10.1029/2004JA010804 (2005).

${ }^{24}$ M. Longmore, S. J. Schwartz, J. Geach, B. M. A. Cooling, I. Dandouras, E. A. Lucek, and A. N. Fazakerley, Ann. Geophys. 23, 3351 (2005).

${ }^{25}$ A. L. La Belle-Hamer, A. Otto, and L. C. Lee, Phys. Plasmas 1, 706 (1994).

${ }^{26}$ H. G. Mitchell, Jr. and J. R. Kan, J. Plasma Phys. 20, 31 (1978).

${ }^{27}$ M. Swisdak, J. F. Drake, M. A. Shay, and B. N. Rogers, J. Geophys. Res. 108, 1218, doi:10.1029/2002JA009726 (2003).

${ }^{28}$ T. D. Phan, J. T. Gosling, G. Paschmann, C. Pasma, J. F. Drake, M. Øieroset, D. Larson, R. P. Lin, and M. S. Davis, Astrophys. J. Lett. 719, L199 (2010).

${ }^{29}$ A. L. La Belle-Hamer, A. Otto, and L. C. Lee, J. Geophys. Res. 100, 11875, doi:10.1029/94JA00969 (1995).

${ }^{30} \mathrm{Q}$. Chen, A. Otto, and L. C. Lee, J. Geophys. Res. 102, 151, doi:10.1029/96JA03144 (1997).

${ }^{31}$ J. H. Li and Z. W. Ma, J. Geophys. Res. 115, A09216, doi:10.1029/ 2010JA015315 (2010).

${ }^{32}$ L. Chacón, D. A. Knoll, and J. M. Finn, Phys. Lett. A 308, 187 (2003).

${ }^{33}$ Q. Q. Shi, Z. Y. Pu, H. Zhang, S. Y. Fu, C. J. Xiao, Q. -G. Zong, T. A. Fritz, and Z. X. Liu, Surv. Geophys. 26, 369 (2005).

${ }^{34}$ V. Roytershteyn and W. Daughton, Phys. Plasmas 15, 082901 (2008).

${ }^{35}$ K. G. Tanaka, M. Fujimoto, and I. Shinohara, Int. J. Geophys. 2010, 202583 (2010).

${ }^{36}$ L. Bettarini, S. Landi, M. Velli, and P. Londrillo, Phys. Plasmas 16, 062302 (2009).

${ }^{37}$ P. A. Cassak, Phys. Plasmas 18, 072106 (2011).

${ }^{38}$ M. A. Shay, J. F. Drake, M. Swisdak, and B. N. Rogers, Phys. Plasmas 11, $2199(2004)$.

${ }^{39}$ A. Otto, J. Geophys. Res. 106, 3751, doi:10.1029/1999JA001005 (2001).

${ }^{40}$ X. L. Chen and P. J. Morrison, Phys. Fluids B 2, 495 (1990). 